Arkivoc

Free to Authors and Readers
A Platinum Open Access Journal for Organic Chemistry

Paper

DOAJ Seal
Arkivoc 2021, part x, 18-26

\title{
Straightforward synthesis of unnatural poly-oxygenated steroid sapogenins
}

\author{
Anielka Rosado-Abón and Martín A. Iglesias-Arteaga* \\ Facultad de Química, Universidad Nacional Autónoma de México, \\ Ciudad Universitaria, 04510 México, D.F., México \\ Email: martin.iglesias@unam.mx
}

Received 07-16-2021

Accepted Manuscript 08-30-2021

Published on line 09-19-2021

\section{Abstract}

Saponins and their biosynthetic intermediates, sapogenins, display a variety of biological activities of interest to the pharmaceutical, cosmetic and food sectors. Three unnatural steroid sapogenins bearing oxygenated functions in rings $A, B$ and $F$ were prepared following a straightforward synthetic protocol that comprises the installation of a carbonyl function in ring $F$, a two-step, one-pot generation of a ketol in $A, B$ rings, and the addition of a cis-diol in ring $A$ by osmylation. Unambiguous NMR characterization is provided.

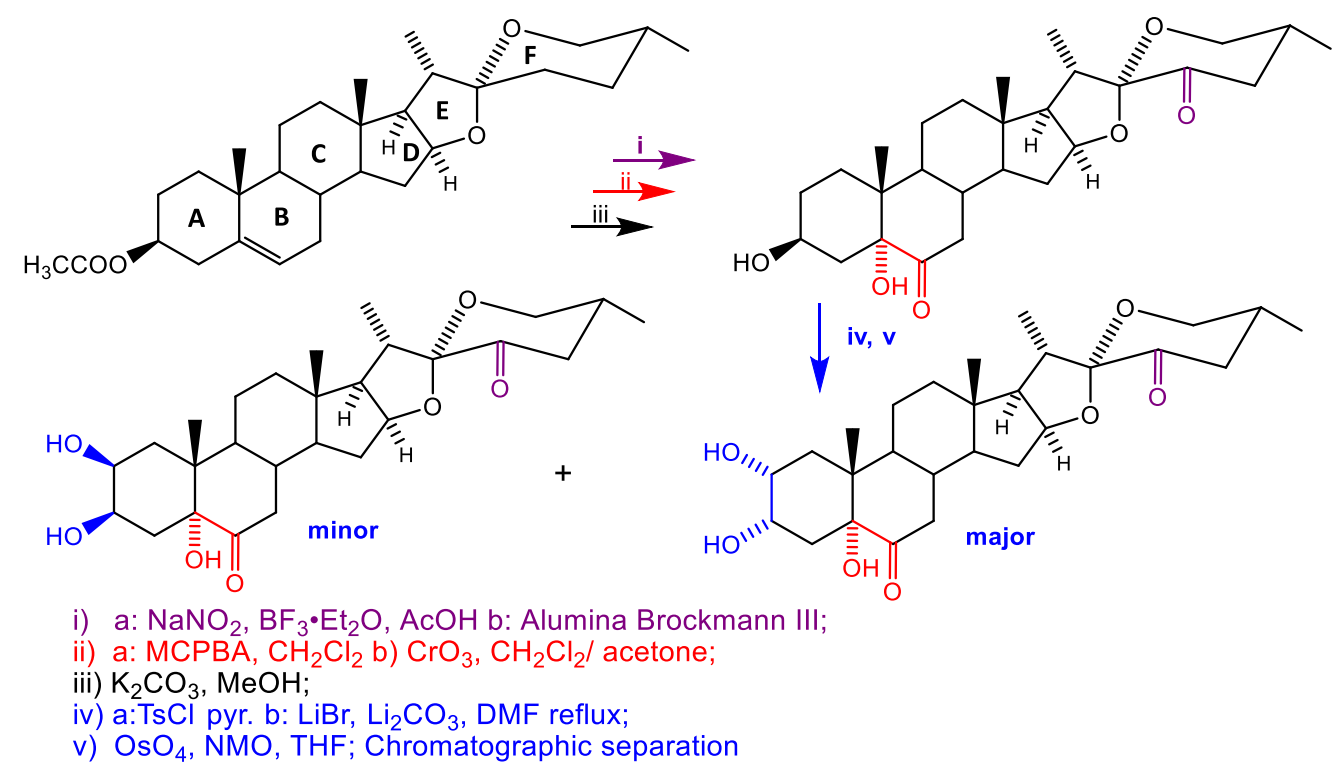

Keywords: Steroid sapogenins, $2 \alpha, 3 \alpha$-diols, $2 \beta, 3 \beta$-diols, $5 \alpha$-hydroxy-6-oxo steroids, Steroid NMR. 


\section{Introduction}

Saponins are secondary metabolites found widespread in nature. A large number of such compounds has been isolated from terrestrial plants as well as from some marine organisms. ${ }^{1-3}$ These kinds of compounds have shown a wide spectrum of properties that include sweet ${ }^{4}$ or bitter taste, ${ }^{5}$ and antimicrobial, insecticidal, and molluscicidal activities ${ }^{6}$ as well as hemolytic ${ }^{7}$ and cytotoxic ${ }^{8}$ properties amongst many others. ${ }^{1,9}$ In addition to the pharmacological and medicinal importance, ${ }^{10}$ the usefulness of saponins in the food industry is well recognised. ${ }^{11}$

Saponins are traditionally classified as triterpene or steroid glycosides according to the aglycone (nonsaccharide) part. The wide diversity of these compounds lays in the structural diversity of the less polar aglycone portion that is coupled to one or more units of different carbohydrates. ${ }^{12}$

In particular, steroid saponins have attracted significant attention since the mid part of the last century due to their utility as raw materials for the production of steroid sapogenins. The aglycone part, obtained by hydrolysis, serves as the classical starting material for the synthesis of steroid hormones ${ }^{13}$ and other biologically active compounds. ${ }^{14-10}$ Additionally, the recognition of biological activity and pharmacological importance of steroid saponins, brought attention of chemists, biochemists and biologists to this family of compounds. Several reviews have covered the isolation, characterization, chemistry, and biological properties of steroid saponins. ${ }^{21-23}$

Consequently, multiple efforts directed towards the synthesis of naturally occurring steroid saponins have been described. ${ }^{24,25}$ Such efforts comprise both the installation of the required functionality in the aglycone part, and synthesis of the carbohydrate building blocks. The last steps of the synthetic sequence are generally the coupling of both units and deprotection of the functional groups.

As a part of our program on the synthesis of biologically active steroids, we have become interested in the synthesis of unnatural steroid sapogenins that could serve as aglycones for the construction of steroid glycosides that may be interesting candidates for biological-activity screening. Herein, we describe the synthesis and characterization of three unnatural steroid sapogenins bearing oxygenated functions in rings $A$, $B$ and $F$.

\section{Results and Discussion}

The synthetic protocol started with the introduction of a carbonyl function at position C-23 of the side chain of diosgenin acetate (1) employing a methodology described by Barton. ${ }^{26}$ According to that report, treatment of a steroid sapogenin (I) with $\mathrm{NaNO}_{2}$ and $\mathrm{BF}_{3} \bullet \mathrm{Et}_{2} \mathrm{O}$ produces a $23 \mathrm{E}$-oximino sapogenin (II) that is hydrolyzed to a 23-keto sapogenin (III) by column chromatography in neutral alumina (Brokmann activity III). Our detailed study of this reaction showed that the compound formed by the treatment with $\mathrm{NaNO}_{2}$ and $\mathrm{BF}_{3} \bullet \mathrm{Et}_{2} \mathrm{O}$ is the 23E-nitroimino derivative (IV) (Scheme 1). ${ }^{27}$ 


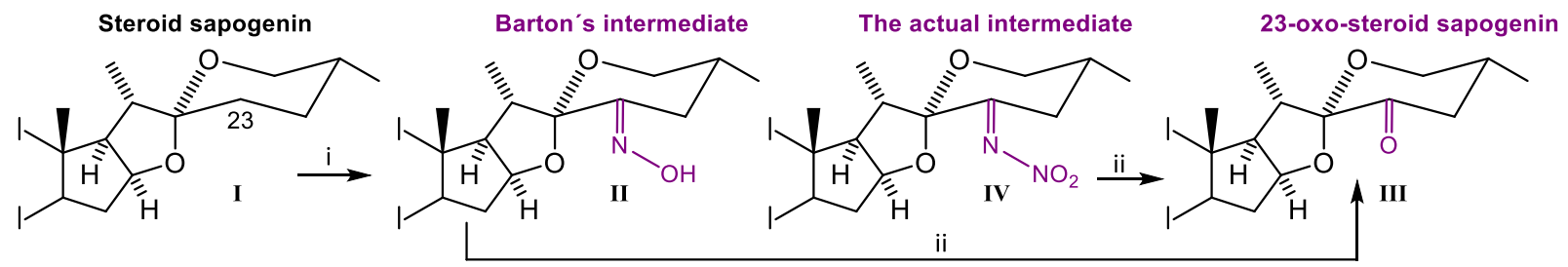

i) $\mathrm{NaNO}_{2}, \mathrm{BF}_{3} \cdot \mathrm{Et}_{2} \mathrm{O}, \mathrm{AcOH}$; ii) Alumina Brockmann III

Scheme 1. Introduction of a carbonyl group at position C-23 of the side chain of steroid sapogenin.

Thus, treatment of diosgenin acetate (1) with $\mathrm{NaNO}_{2}$ and $\mathrm{BF}_{3} \bullet \mathrm{Et}_{2} \mathrm{O}$ in acetic acid followed by column chromatography in neutral alumina (Brokmann activity III) following Barton's procedure afforded the ketone $\mathbf{2}$ that was subsequently converted into the acetylated ketol 3 employing our simple one-pot procedure which includes epoxidation with meta-chloroperoxybenzoic acid (mCPBA) followed by oxidative cleavage of the oxirane ring with $\mathrm{CrO}_{3}{ }^{28}$

Hydrolysis of the acetate at position C-3 afforded the target dihydroxylated ketone 4 that was converted into the unsaturated ketol 5 by tosylation in pyridine followed by elimination using treatment with $\mathrm{LiBr}$ and $\mathrm{Li}_{2} \mathrm{CO}_{3}$ in refluxing DMF. Subsequent dihydroxylation employing $\mathrm{OsO}_{4}$ and $\mathrm{N}$-methylmorpholine $\mathrm{N}$-oxide (NMO) as co-oxidant in TMF afforded a mixture of the target trihydroxylated diketone 6 as the maJor compound, accompanied by its $2 \beta, 3 \beta$-diastereomer 7 that were separated by column chromatography (Scheme 2).
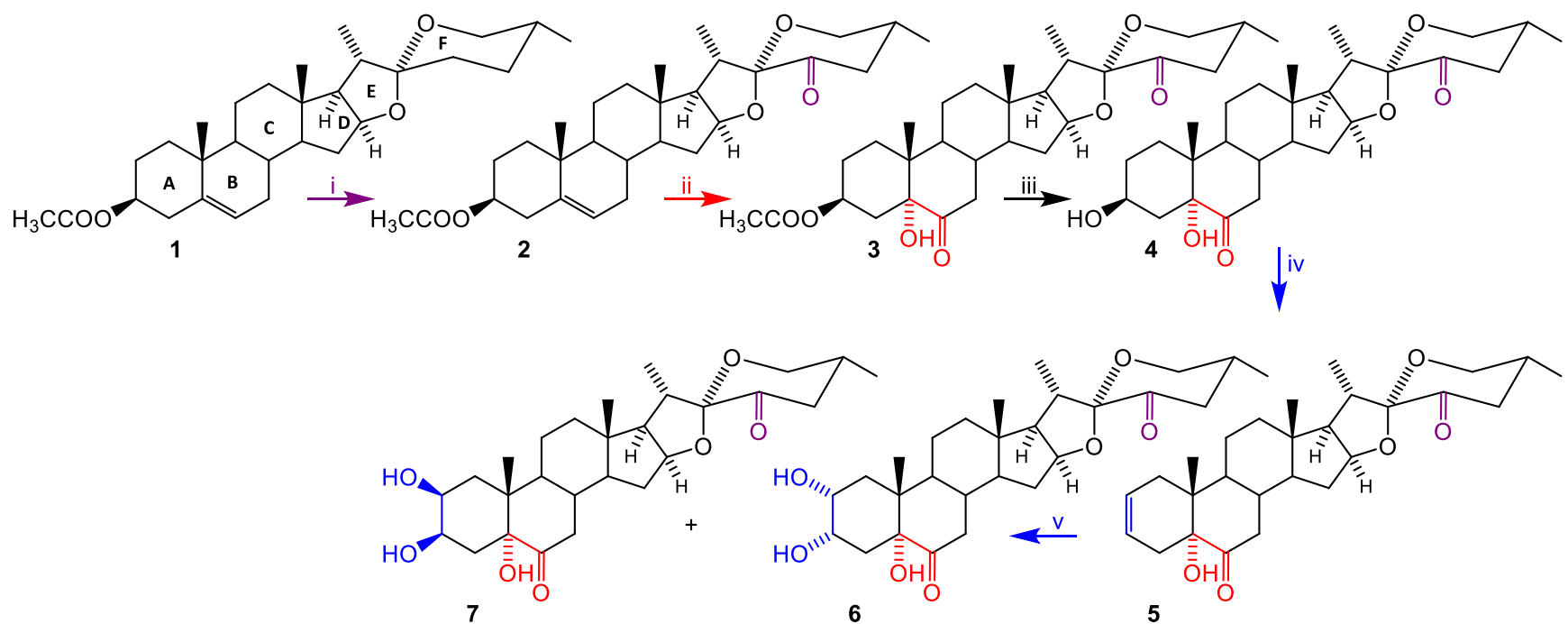

i) a: $\mathrm{NaNO}_{2}, \mathrm{BF}_{3} \cdot \mathrm{Et}_{2} \mathrm{O}, \mathrm{AcOH}$; b: Alumina Brockmann III; ii) a: $\mathrm{MCPBA}, \mathrm{CH}_{2} \mathrm{Cl}_{2}$; b: $\mathrm{CrO}_{3}, \mathrm{CH}_{2} \mathrm{Cl}_{2} /$ acetone; iii) $\mathrm{K}_{2} \mathrm{CO}_{3}, \mathrm{MeOH}$;

iv) a: $\mathrm{TsCl}$ pyr. b: $\mathrm{LiBr}, \mathrm{Li}_{2} \mathrm{CO}_{3}$, DMF reflux; v) $\mathrm{OsO}_{4}, \mathrm{NMO}$, THF; Chromatographic separation

Scheme 2. Synthesis of the polyoxygenated steroid sapogenins 4,6 and 7 .

NMR signals assignments (Tables 1 and 2) were carried out with the aid of a combination of 1D and 2D NMR techniques that included ${ }^{1} \mathrm{H},{ }^{13} \mathrm{C}, 1 \mathrm{H}-1 \mathrm{H}$ COSY, Nuclear Overhauser Effect Spectroscopy (NOESY), Heteronuclear Single Quantum Correlation (HSQC) and Heteronuclear Multiple Bond Correlation (HMBC). The observed NOEs allowed the determination of the orientations of the $2 \alpha, 3 \alpha$ and $2 \beta, 3 \beta$ diols introduced into the $A$ rings of compounds 6 and 7 , respectively (Figure 1). 
Table 1. Selected ${ }^{1} \mathrm{H}$ signals of steroid sapogenins 4,6 and $7 . \delta(p p m)$ and multiplicities*

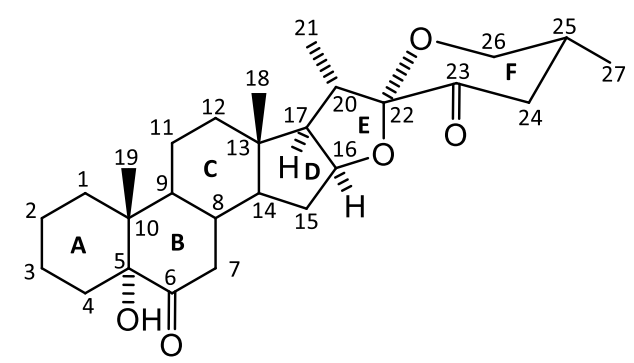

\begin{tabular}{cccc}
\hline & $\mathbf{4}$ & $\mathbf{6}$ & $\mathbf{7}$ \\
\hline $\mathbf{H}-2$ & n.a. & $3.64 \mathrm{~m}$ & $3.79-3.71 \mathrm{~m}$ \\
OH (C-2) & - & $4.55 \mathrm{~m}$ & $4.09 \mathrm{~d}$ \\
H-3 & $3.96 \mathrm{~m}$ & $3.92 \mathrm{~m}$ & $3.79-3.71 \mathrm{~m}$ \\
OH (C-3) & n.a. & $5.48 \mathrm{~d}$ & $4.29 \mathrm{~d}$ \\
OH (C-5) & $2.95 \mathrm{~s}$ & $5.65 \mathrm{~s}$ & $5.38 \mathrm{~s}$ \\
H-16 & $4.60 \mathrm{dt}$ & $4.55 \mathrm{~m}$ & $4.55 \mathrm{dt}$ \\
H-18 & $0.75 \mathrm{~s}$ & $0.68 \mathrm{~s}$ & $0.67 \mathrm{~s}$ \\
H-19 & $0.79 \mathrm{~s}$ & $0.68 \mathrm{~s}$ & $0.88 \mathrm{~s}$ \\
H-21 & $0.93 \mathrm{~d}$ & $0.86 \mathrm{~d}$ & $0.86 \mathrm{~d}$ \\
H-26 ax & $3.77 \mathrm{dd}$ & $3.64 \mathrm{~m}$ & $3.65 \mathrm{dd}$ \\
H-26 eq. & $3.58 \mathrm{dd}$ & $3.64 \mathrm{~m}$ & $3.59 \mathrm{ddd}$ \\
H-27 & $0.93 \mathrm{~d}$ & $0.87 \mathrm{~d}$ & $0.88 \mathrm{~d}$ \\
\hline
\end{tabular}

* Solvents: $\mathrm{CDCl}_{3}$ for 4; DMSO- $\mathrm{d}_{6}$ for 6 and 7. NA not assigned.

Table 2. Selected ${ }^{13} \mathrm{C}$ NMR signals of steroid sapogenins 4,6 and 7. $\delta(\mathrm{ppm}){ }^{*}$

\begin{tabular}{cccc}
\hline & $\mathbf{4}$ & $\mathbf{6}$ & $\mathbf{7}$ \\
\hline C-2 & 30.3 & 66.5 & 68.3 \\
C-3 & 67.2 & 69.3 & 67.1 \\
C-5 & 80.4 & 79.1 & 79.3 \\
C-6 & 212.7 & 210.2 & 212.1 \\
C-16 & 83.0 & 82.6 & 82.6 \\
C-18 & 16.1 & 15.9 & 16.0 \\
C-19 & 14.1 & 14.5 & 15.8 \\
C-21 & 14.3 & 14.4 & 14.4 \\
C-22 & 109.8 & 109.1 & 109.1 \\
C-23 & 201.7 & 201.4 & 201.4 \\
C-26 & 65.7 & 64.9 & 64.9 \\
C-27 & 17.1 & 16.7 & 16.7
\end{tabular}

* Solvents: $\mathrm{CDCl}_{3}$ for 4; DMSO- $\mathrm{d}_{6}$ for 6 and 7. 


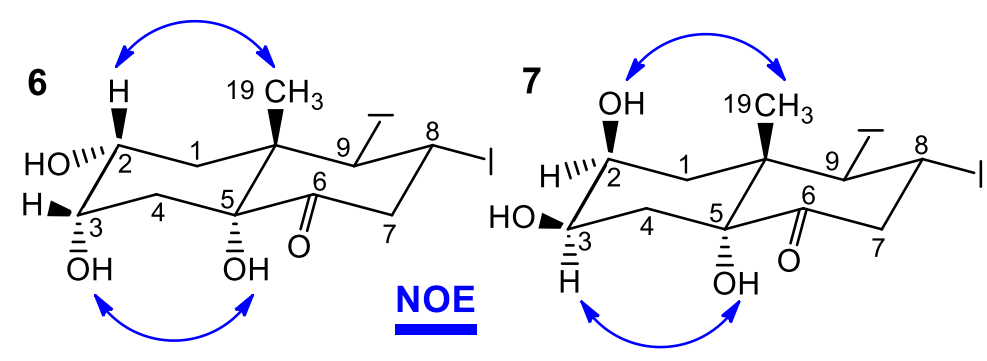

Figure 1. Observed NOEs in compounds 6 and 7.

\section{Conclusions}

We have set up procedures for the synthesis and unambiguous characterization of unnatural steroid sapogenins that will serve as aglycones for the construction of steroid glycosides which may be interesting candidates for biological-activity screening.

\section{Experimental Section}

General. Reactions were monitored by TLC on ALUGRAM® SIL G/UV254 plates from MACHEREY-NAGEL. Chromatographic plates were sprayed with a $1 \%$ solution of vanillin in $50 \% \mathrm{HClO} 4$ and heated until color developed. Melting points were measured on a Melt-Temp II apparatus. NMR spectra were recorded in $\mathrm{CDCl}_{3}$ or DMSO- $\mathrm{d}_{6}$ solutions on a Varian INOVA 400 spectrometer using the solvents' signals $\left(\mathrm{CDCl}_{3} ; 7.26 \mathrm{ppm}\right.$ for ${ }^{1} \mathrm{H}$ and $77.00 \mathrm{ppm}$ for ${ }^{13} \mathrm{C}$ ) or $\left(\mathrm{DMSO}-\mathrm{d}_{6}, 2.50 \mathrm{ppm}\right.$ for ${ }^{1} \mathrm{H}$ and $39.52 \mathrm{ppm}$ for ${ }^{13} \mathrm{C}$ ) as references. All 2D NMR spectra were recorded using the standard pulse sequences and parameters recommended by the manufacturer and were processed employing the MestreNova NMR program [See http://mestrelab.com/]. Mass spectra (EI) were registered on a Thermo-Electron spectrometer model DFS (Double Focus Sector). HRMS spectra were registered using a PERKIN ELMER Model: AxION-2 TOF MS spectrometer.

(25R)-3ß-acetoxyspiros-5-en-23-one (2). $\mathrm{NaNO}_{2}(0.5256 \mathrm{~g})$ was slowly added (45 min) to a solution of diosgenin acetate $(\mathbf{1})(3 \mathrm{~g}, 6.57 \mathrm{mmol})$ and $\mathrm{BF}_{3} . \mathrm{Et}_{2} \mathrm{O}(2.69 \mathrm{~mL})$ glacial in acetic acid $(75 \mathrm{~mL})$. After conclusion of this addition, additions of $\mathrm{BF}_{3} . \mathrm{Et}_{2} \mathrm{O}(2.69 \mathrm{~mL})$ and $\mathrm{NaNO}_{2}(0.5256 \mathrm{~g}$ in $45 \mathrm{~min}$.) were repeated, and the resulting mixture was stirred for 1 hour and then poured into ice/water. The resulting solid was filtered off, washed with abundant water, and dissolved in ethyl acetate $(200 \mathrm{~mL})$. The organic solution was washed with a $5 \%$ aqueous solution of $\mathrm{Na}_{2} \mathrm{CO}_{3}(2 \times 50 \mathrm{~mL}), \mathrm{H}_{2} \mathrm{O}(1 \times 50 \mathrm{~mL})$ and $\mathrm{NaCl}(1 \times 50 \mathrm{~mL})$, dried $\left(\right.$ anh. $\left.\mathrm{Na}_{2} \mathrm{SO}_{4}\right)$ and evaporated. The resulting syrup was dissolved in the smallest amount of a $1 / 1$ benzene/hexane solution and left to stand overnight in a chromatographic column packed with $\mathrm{Al}_{2} \mathrm{O}_{3}$ (Brockman activity III). Elution with a

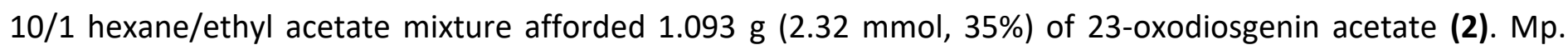
190-192 으 (from ethyl acetate/hexane). Lit. ${ }^{29} 191-192.5^{\circ} \mathrm{C} .{ }^{1} \mathrm{H} \mathrm{NMR}\left(400 \mathrm{MHz}, \mathrm{CDCl}_{3}\right) \delta \mathrm{ppm} 5.35$ (d, J $5.1 \mathrm{~Hz}$, $1 \mathrm{H}, \mathrm{H}-6), 4.64-4.54(\mathrm{~m}, 2 \mathrm{H}, \mathrm{H}-16, \mathrm{H}-3), 3.78$ (dd, J 11.3, $11.3 \mathrm{~Hz}, 1 \mathrm{H}, \mathrm{H}-26$ ax.), 3.58 (ddd, J 11.2, 4.6, 1.4 Hz, $1 \mathrm{H}, \mathrm{H}-26$ eq. ), 2.87 (dd, J 13.7, $6.9 \mathrm{~Hz}, 1 \mathrm{H}, \mathrm{H}-20), 2.49-2.38(\mathrm{~m}, 2 \mathrm{H}, \mathrm{H}-24), 2.36-2.21(\mathrm{~m}, 3 \mathrm{H}, \mathrm{H}-4, \mathrm{H}-25), 2.02$ (s, 3H, $\mathrm{CH}_{3}$ acetyl), 1.02 (s, 3H, H-19), 0.93 (d, J $\left.7.1 \mathrm{~Hz}, 3 \mathrm{H}, \mathrm{H}-21\right), 0.93(\mathrm{~d}, J 6.5 \mathrm{~Hz}, 3 \mathrm{H}, \mathrm{H}-27), 0.78$ (s, 3H, H18). ${ }^{13} \mathrm{C}$ NMR (100.53 MHz) $\delta$ ppm $36.9 \mathrm{C}-1,27.7 \mathrm{C}-2,73.8 \mathrm{C}-3,38.1 \mathrm{C}-4,139.8 \mathrm{C}-5,122.2 \mathrm{C}-6,32.0 \mathrm{C}-7,31.4 \mathrm{C}-$ 8, 49.9 C-9, 36.7 C-10, 20.7 C-11, 39.5 C-12, 40.7 C-13, 56.5 C-14, 31.8 C-15, 83.3 C-16, 61.7 C-17, 16.0 C-18, 
19.3 C-19, 34.8 C-20, 14.4 C-21, 109.8 C-22, 201.8 C-23, 45.3 C-24, 35.8 C-25, 65.6 C-26, 17.1 C-27, 170.5 C=0 acetyl, $21.4 \mathrm{CH}_{3}$ acetyl.

(25R)-3 $\beta$-acetoxy-5-hydroxy-5 $\alpha$-spirostan-6,23-dione (3). meta-Chloroperoxybenzoic acid (mCPBA) (414.5 $\mathrm{mg}$ ) was added to a solution of the ketone $2(798.4 \mathrm{mg}, 1.70 \mathrm{mmol})$ in $\mathrm{CH}_{2} \mathrm{Cl}_{2}(10 \mathrm{~mL})$ and the mixture was stirred until the starting material disappeared $(1 \mathrm{~h}, \mathrm{TLC})$. Acetone $(20 \mathrm{~mL})$ was added, and the mixture was cooled to $0^{\circ} \mathrm{C}$ in an ice bath before the dropwise addition of a solution of $\mathrm{CrO}_{3}(303.4 \mathrm{mg})$ in water $(1 \mathrm{~mL})$. The ice bath was removed, the mixture was stirred at room temperature for $20 \mathrm{~min}$ and cooled to $0^{\circ} \mathrm{C}$ in the ice bath prior to dropwise addition of a solution of $\mathrm{CrO}_{3}(773.6 \mathrm{mg})$ in water $(2.3 \mathrm{~mL})$. The ice bath was removed, and the mixture stirred for $2.5 \mathrm{~h}$, before addition of water $(20 \mathrm{~mL})$ and extraction with ethyl acetate $(2 \times 25$ $\mathrm{mL})$. The organic layer was washed sequentially with water $(10 \times 20 \mathrm{~mL})$, a $10 \% \mathrm{NaHCO}_{3}$ solution $(5 \times 20 \mathrm{~mL})$, water $(2 \times 20 \mathrm{~mL})$ and brine $(20 \mathrm{~mL})$, then dried (anh. $\left.\mathrm{Na}_{2} \mathrm{SO}_{4}\right)$ and evaporated to afford $787.6 \mathrm{mg}(1.57 \mathrm{mmol}$, $92 \%$ ) of the desired ketol 3. Mp. $272-274{ }^{\circ} \mathrm{C}$ (from ethyl acetate/hexane). ${ }^{1} \mathrm{H} \mathrm{NMR}(400 \mathrm{MHz}, \mathrm{CDCl} 3) \delta \mathrm{ppm}$ $5.05-4.95(\mathrm{~m}, 1 \mathrm{H}, \mathrm{H}-3), 4.61$ (td, J 7.7, $6.0 \mathrm{~Hz}, 1 \mathrm{H}, \mathrm{H}-16), 3.76$ (dd, J 11.3, $11.3 \mathrm{~Hz}, 1 \mathrm{H}, \mathrm{H}-26 \mathrm{ax}$ ), 3.58 (ddd, J 11.2, 4.1, $1.2 \mathrm{~Hz}, 1 \mathrm{H}, \mathrm{H}-26$ eq. ), 3.01 (s, 1H, OH-5), $2.91-2.83(\mathrm{~m}, 1 \mathrm{H}, \mathrm{H}-20), 2.76$ (t, J $12.3 \mathrm{~Hz}, 1 \mathrm{H}, \mathrm{H}-7 \mathrm{ax}$.$) ,$ $2.45-2.41(\mathrm{~m}, 2 \mathrm{H}, \mathrm{H}-24), 2.33-2.23(\mathrm{~m}, 1 \mathrm{H}, \mathrm{H}-25), 2.10$ (dd, J 13.0, $4.0 \mathrm{~Hz}, 1 \mathrm{H}, \mathrm{H}-7$ eq. ), 1.99 (s, 3H, s, 3H, $\mathrm{CH}_{3}$ acetyl), $0.93(\mathrm{~d}, J 7.5 \mathrm{~Hz}, 3 \mathrm{H}, \mathrm{H}-21), 0.93(\mathrm{~d}, J 6.4 \mathrm{~Hz}, 3 \mathrm{H}, \mathrm{H}-27), 0.81(\mathrm{~s}, 3 \mathrm{H}, \mathrm{H}-19), 0.75(\mathrm{~s}, 3 \mathrm{H}, \mathrm{H}-18) .{ }^{13} \mathrm{C}$ NMR (100.53 MHz) $\delta$ ppm 29.5 C-1, 26.2 C-2, 70.5 C-3, 32.4 C-4, 80.2 C-5, 211.8 C-6, 41.7 C-7, 36.7 C-8, 44.3 C9, 42.4 C-10, 21.1 C-11, 39.3 C-12, 41.6 C-13, 56.1 C-14, 31.5 C-15, 83.0 C-16, 61.7 C-17, 16.1 C-18, 14.0 C-19, 34.7 C-20, 14.3 C-21, 109.8 C-22, 201.7 C-23, 45.2 C-24, 35.8 C-25, 65.7 C-26, 17.1 C-27, 171.0 C=0 acetyl, 21.3 $\mathrm{CH}_{3}$ acetyl. MS (IE, 70 e-V): $502 \mathrm{M}^{+}, 475,474,430,421,420,419,360,359,358,345,341,327,313,296,285$, 267(100\%), 249, 239, 209, 185, 175, 173, 149, 147, 133, 121, 105, 93, 91, 79, 56. HRMS (EI) observed 502.2897, calculated for $\mathrm{C}_{29} \mathrm{H}_{42} \mathrm{O}_{7} 502.2925$.

(25R)-3 $\beta, 5$-dihydroxy-5 $\alpha$-spirostan-6,23-dione (4). A suspension of the acetylated diketone 3 (699.6 mg, 1.39 $\mathrm{mmol}$ ) in a saturated solution of $\mathrm{K}_{2} \mathrm{CO}_{3}$ in methanol $(30 \mathrm{~mL})$ was stirred overnight. Half of the solvent was evaporated off and the remaining mixture was poured into ice/water. The obtained solid was filtered off, washed with abundant water, and dried by air to afford $568.5 \mathrm{mg}(1.23 \mathrm{mmol}, 89 \%)$ of the dihydroxylated diketone 4. Mp. $296-298{ }^{\circ} \mathrm{C}$ (ethyl acetate/hexane). ${ }^{1} \mathrm{H} \mathrm{NMR}\left(400 \mathrm{MHz}, \mathrm{CDCl}_{3}\right) \delta \mathrm{ppm} 4.60(\mathrm{dt}, J 7.5,6.1 \mathrm{~Hz}$, $1 \mathrm{H}, \mathrm{H}-16), 3.96$ (m, 1H, H-3), 3.77 (dd, J 11.3, $11.3 \mathrm{~Hz}, 1 \mathrm{H}, \mathrm{H}-26$ ax.), 3.58 (dd, J 11.1, $4.0 \mathrm{~Hz}, 1 \mathrm{H}, \mathrm{H}-26$ eq. ), 2.95 (a, 1H, OH-5), $2.92-2.83(\mathrm{~m}, 1 \mathrm{H}, \mathrm{H}-20), 2.75$ (t, J $12.1 \mathrm{~Hz}, 1 \mathrm{H}, \mathrm{H}-7 \mathrm{ax}.), 2.51-2.41(\mathrm{~m}, 2 \mathrm{H}, \mathrm{H}-24), 2.33-$ 2.22 (m, 1H, H-25), 2.09 (dd, J 13.1, $2.5 \mathrm{~Hz}, 1 \mathrm{H}, \mathrm{H}-7$ eq. ), 0.93 (d, J $6.7 \mathrm{~Hz}, 6 \mathrm{H}, \mathrm{H}-21$ and H-27), 0.79 (s, 3H, H19), 0.75 (s, 3H, H-18). ${ }^{13} \mathrm{C}$ NMR (100.53 MHz) $\delta$ ppm 29.8 C-1, 30.3 C-2, 67.2 C-3, 36.1 C-4, 80.4 C-5, 212.7 C-6, 41.8 C-7, 36.7 C-8, 44.4 C-9, 42.4 C-10, 21.2 C-11, 39.3 C-12, 41.6 C-13, 56.2 C-14, 31.5 C-15, 83.0 C-16, 61.6 C17, 16.1 C-18, 14.1 C-19, 34.7 C-20, 14.3 C-21, 109.8 C-22, 201.7 C-23, 45.2 C-24, 35.8 C-25, 65.7 C-26, 17.1 C27. MS (IE, 70 e-V): $460 \mathrm{M}^{+}, 433,432,414,379,378,377,359,341,323,313,303,285,267$ (100\%), 249, 239, $225,199,185,175,173,165,147,121,107,93,91,81,67,56$. HRMS (EI): observed 460.2828, calculated for $\mathrm{C}_{27} \mathrm{H}_{40} \mathrm{O}_{6} 460.2819$.

(25R)-5-Hydroxy-5 $\alpha$-espirost-2-en-6,23-dione (5). Tosyl chloride (672 mg) was added to a solution of the dihydroxylated diketone $4(638.3 \mathrm{mg}, 1.39 \mathrm{mmol})$ in dry pyridine $(8 \mathrm{~mL})$ and the mixture was stirred for $24 \mathrm{~h}$ before pouring into a $3 \% \mathrm{HCl} /$ ice bath. The produced solid was filtered off and dissolved in ethyl acetate $(60$ $\mathrm{mL}$ ), and the organic solution was washed with water $(2 \times 20 \mathrm{~mL})$, dried (anh. $\left.\mathrm{Na}_{2} \mathrm{SO}_{4}\right)$ and evaporated. The residue was stirred with $\mathrm{LiBr}(713 \mathrm{mg})$ and $\mathrm{Li}_{2} \mathrm{CO}_{3}(590 \mathrm{mg})$ under reflux in dry DMF $(12 \mathrm{~mL})$ for $3 \mathrm{~h}$ and the mixture was cooled to room temperature. Ethyl acetate $(60 \mathrm{~mL})$ was added, the mixture was filtered, the inorganic salts were washed with ethyl acetate $(4 \times 5 \mathrm{~mL})$ and the organic solution was washed with water $(6 \times$ $20 \mathrm{~mL}$ ) dried (anh. $\mathrm{Na}_{2} \mathrm{SO}_{4}$ ) and evaporated. The produced residue was purified in a chromatographic column 
packed with silica gel employing hexane-ethyl acetate as eluent to afford $383.6 \mathrm{mg}(0.87 \mathrm{mmol}, 63 \%)$ of the desired unsaturated ketol 5. Mp. $258-260{ }^{\circ} \mathrm{C}$ (from hexane/ethyl acetate). ${ }^{1} \mathrm{H} \mathrm{NMR}\left(400 \mathrm{MHz}, \mathrm{CDCl}_{3}\right) \delta \mathrm{ppm}$ $5.69-5.57(\mathrm{~m}, 2 \mathrm{H}, \mathrm{H}-2$ and H-3), $4.65-4.57(\mathrm{~m}, 1 \mathrm{H}, \mathrm{H}-16), 3.77(\mathrm{dd}, J 11.3,11.3 \mathrm{~Hz}, 1 \mathrm{H}, \mathrm{H}-26 \mathrm{ax}$ ) , 3.57 (ddd, J 11.1, 4.4, $1.1 \mathrm{~Hz}, 1 \mathrm{H}, \mathrm{H}-26$ eq. ), 2.88 (q, J $6.8 \mathrm{~Hz}, 1 \mathrm{H}, \mathrm{H}-20), 2.69$ (t, J $12.3 \mathrm{~Hz}, 1 \mathrm{H}, \mathrm{H}-7 \mathrm{ax}.), 2.61$ (dd, J $4.2,2.1$ $\mathrm{Hz}, 1 \mathrm{H}, \mathrm{H}-1), 2.46-2.41(\mathrm{~m}, 1 \mathrm{H}, \mathrm{H}-24), 2.34-2.21$ (m, 1H, H-25), 2.19 (dd, J 12.7, $3.6 \mathrm{~Hz}, 1 \mathrm{H}, \mathrm{H}-7$ eq. ), 2.12 (dd, J 15.9, $2.1 \mathrm{~Hz}, 1 \mathrm{H}, \mathrm{H}-4), 0.93$ (d, J $7.3 \mathrm{~Hz}, 3 \mathrm{H}, \mathrm{H}-21), 0.92$ (d, J $6.4 \mathrm{~Hz}, 3 \mathrm{H}, \mathrm{H}-27), 0.75$ (s, 3H, H-18), 0.71 (s, 3H, H-19). ${ }^{13} \mathrm{C}$ NMR (100.53 MHz) $\delta$ ppm $30.1 \mathrm{C}-1,125.4 \mathrm{C}-2,122.3 \mathrm{C}-3,34.5 \mathrm{C}-4,77.9 \mathrm{C}-5,210.7 \mathrm{C}-6,42.6$ C-7, 36.8 C-8, 45.1 C-9, 42.2 C-10, 20.7 C-11, 39.3 C-12, 41.3 C-13, 56.2 C-14, 31.4 C-15, 82.9 C-16, 61.6 C-17, 16.0 C-18, 14.6 C-19, 34.7 C-20, 14.3 C-21, 109.8 C-22, 201.7 C-23, 45.2 C-24, 35.8 C-25, 65.7 C-26, 17.0 C-27. MS (FAB): $443[\mathrm{MH}]^{+}, 359,341,285,259,207,159,149,133,105,91,83,57$. HRMS (FAB): Found 443.2811, calculated for $\mathrm{C}_{27} \mathrm{H}_{39} \mathrm{O}_{5} 443.2792[\mathrm{MH}]^{+}$.

(25R)-2 $\alpha, 3 \alpha, 5$-Trihydroxy-5 $\alpha$-spirostan-6,23-dione (6) and (25R)-2 $\beta, 3 \beta, 5$-trihydroxy-5 $\alpha$-spirostan-6,23-dione (7). $\mathrm{N}$-methylmorpholine $\mathrm{N}$-oxide $(544 \mathrm{mg})$ and a $12.5 \mathrm{mg} / \mathrm{mL}$ solution of $\mathrm{OsO}_{4}(3.26 \mathrm{~mL})$ in tert-butyl alcohol were added to a solution of the unsaturated ketol $5(301.0 \mathrm{mg}, 0.68 \mathrm{mmol})$ in THF $(7 \mathrm{~mL})$ and the mixture was stirred under $\mathrm{N}_{2}$ for $24 \mathrm{~h}$ before addition of a solution of $\mathrm{Na}_{2} \mathrm{SO}_{3}$ ( $246 \mathrm{mg}$ ) in water $(2 \mathrm{~mL})$. The resulting mixture was stirred for $90 \mathrm{~min}$, ethyl acetate $(80 \mathrm{~mL})$ was added, the organic solution was washed with brine $\left(5 \times 15 \mathrm{~mL}\right.$ ), dried (anh. $\left.\mathrm{Na}_{2} \mathrm{SO}_{4}\right)$ and evaporated. The produced residue was purified in a chromatographic column packed with silica gel, employing $1 / 1$ hexane/ethyl acetate mixture to afford the epimeric trihydroxylated diketones 6 and 7.

(25R)-2 $\alpha, 3 \alpha, 5-T r i h y d r o x y-5 \alpha$-spirostan-6,23-dione (6). Yield $132.7 \mathrm{mg}(0.28 \mathrm{mmol}, 41 \%) . \mathrm{Mp} .286-288{ }^{\circ} \mathrm{C}$ (from acetone). ${ }^{1} \mathrm{H}$ NMR (400 MHz, DMSO-d6) $\delta$ ppm $5.65(\mathrm{~s}, 1 \mathrm{H}, \mathrm{OH}-5), 5.48$ (d, J $\left.3.2 \mathrm{~Hz}, 1 \mathrm{H}, \mathrm{OH}-3\right), 4.55(\mathrm{~m}$, 2H, H-16, OH-2), 3.92 (a, 1H, H-3), 3.64 (m, 3H, H-2, H-26 ax., eq. ), 2.56 (t, J 12.3 Hz, 1H, H-7ax.), 0.87 (d, J 6.2 $\mathrm{Hz}, 3 \mathrm{H}, \mathrm{H}-27), 0.86$ (d, J $6.8 \mathrm{~Hz}, 3 \mathrm{H}, \mathrm{H}-21), 0.68$ (s, 3H, H-19), 0.68 (s, 3H, H-18). ${ }^{13} \mathrm{C} \mathrm{NMR}(100.53 \mathrm{MHz})$ ? ppm 30.5 C-1, 66.5 C-2, 69.3 C-3, 34.5 C-4, 79.1 C-5, 210.2 C-6, 41.0 C-7, 35.9 C-8, 44.2 C-9, 44.7 C-10, 20.5 C-11, 38.9 C-12, 41.0 C-13, 55.5 C-14, 31.0 C-15, 82.6 C-16, 60.8 C-17, 15.9 C-18, 14.5 C-19, 34.3 C-20, 14.4 C-21, 109.1 C-22, 201.4 C-23, 44.7 C-24, 35.3 C-25, 64.9 C-26, 16.7 C-27. MS (FAB): 477 [M+1] $]^{+}, 459,385,341,327$, 281, 267, 221, 207, 165, 147, 136, 97, 91, 73, 69, 57 (100\%). HRMS (FAB): Found 477.2872, calculated for $\mathrm{C}_{27} \mathrm{H}_{41} \mathrm{O}_{7} 477.2847[\mathrm{MH}]^{+}$.

(25R)-2 $\beta, 3 \beta, 5$-Trihydroxy-5 $\alpha$-spirostan-6,23-dione (7). Yield $64.5 \mathrm{mg}(0.14 \mathrm{mmol}, 21 \%)$. Mp. $288-290{ }^{\circ} \mathrm{C}$ (from acetone/water). ${ }^{1} \mathrm{H}$ NMR (400 MHz, DMSO-d6) $\delta$ ppm $5.38(\mathrm{~s}, 1 \mathrm{H}, \mathrm{OH}-5), 4.55$ (dt, J 7.6, $\left.6.4 \mathrm{~Hz}, 1 \mathrm{H}, 1 \mathrm{H}, \mathrm{H}-16\right)$, $4.29(\mathrm{~d}, J 6.4 \mathrm{~Hz}, 1 \mathrm{H}, \mathrm{OH}-3), 4.09$ (d, J $2.2 \mathrm{~Hz}, 1 \mathrm{H}, \mathrm{OH}-2), 3.79-3.71$ (m, 2H, H-2 and H-3), 3.65 (dd, J 11.1 , $11.1 \mathrm{~Hz}, 1 \mathrm{H}, \mathrm{H}-26$ ax.), 3.59 (ddd, J 11.1, 5.0, $1.2 \mathrm{~Hz}, 1 \mathrm{H}, \mathrm{H}-26$ eq. ), 2.75 (q, J $6.9 \mathrm{~Hz}, 1 \mathrm{H}, \mathrm{H}-20), 2.67$ (t, J 12.3 $\mathrm{Hz}, 1 \mathrm{H}, \mathrm{H}-7 \mathrm{ax}.), 2.43-2.30(\mathrm{~m}, 2 \mathrm{H}, \mathrm{H}-24), 2.18$ (qd, J 11.6, $5.4 \mathrm{~Hz}, 1 \mathrm{H}, \mathrm{H}-8), 0.88(\mathrm{~s}, 3 \mathrm{H}, \mathrm{H}-19), 0.88(\mathrm{~d}, J 6.5$ $\mathrm{Hz}, 3 \mathrm{H}, \mathrm{H}-27), 0.86$ (d, J $7.1 \mathrm{~Hz}, 3 \mathrm{H}, \mathrm{H}-21), 0.67$ (s, 3H, H-18). ${ }^{13} \mathrm{C}$ NMR (100.53 MHz) $\delta \mathrm{ppm} \mathrm{30.5} \mathrm{C-1,} \mathrm{68.3} \mathrm{C-}$ 2, 67.1 C-3, 36.6 C-4, 79.3 C-5, 212.1 C-6, 41.2 C-7, 35.3 C-8, 44.1 C-9, 41.5 C-10, 20.8 C-11, 39.0 C-12, 41.1 C13, 55.5 C-14, 31.1 C-15, 82.6 C-16, 60.9 C-17, 16.0 C-18, 15.8 C-19, 34.2 C-20, 14.4 C-21, 109.1 C-22, 201.4 C23, 44.7 C-24, 35.4 C-25, 64.9 C-26, 16.7 C-27. Elemental analysis: Found C $68.34 \%$ H $8.54 \%$, calculated for $\mathrm{C}_{27} \mathrm{H}_{40} \mathrm{O}_{7} \mathrm{C} 68.04 \% \mathrm{H} 8.46 \%$.

\section{Acknowledgements}

The authors thank the financial support provided by Dirección General de Asuntos del Personal Académico (Project DGAPA-IN218820), Consejo Nacional de Ciencia y Tecnología (Project A1-S-8019), and Facultad de 
Química, Universidad Nacional Autónoma de México (PAIP-5000-9063). Thanks are due also to Nayeli Balbiaux, Rosa I. del Villar and Jessica Amacosta (USAll-FQ) for registering NMR and MS spectra.

\section{Supplementary Material}

Copies of the ${ }^{1} \mathrm{H}$ NMR and ${ }^{13} \mathrm{CNMR}$ spectra of compounds 2-7 are provided in the online edition of the text.

\section{References}

1. Vincken, J.P.; Heng, L.; de Groot, A.; Gruppen, H. Phytochemistry 2007, 68, 275-297.

https://doi.org/10.1016/j.phytochem.2006.10.008

2. Cheok, C. Y.; Salam, H. A. K.; Sulaiman, R. Food Res. Int. 2014, 59, 16-40.

https://doi.org/10.1016/j.foodres.2014.01.057

3. El Aziz, M. M. A.; Ashour, A. S.; Melad, A. S. G. J. Nanomed. Res. 2019, 7(4), 282-288.

https://doi.org/10.15406/jnmr.2019.07.00199

4. Grenby, T. H. Trends Food Sci. Technol. 1991, 2, 2-6.

https://doi.org/10.1016/0924-2244(91)90598-D

5. Heng, L.; Vincken, J.-P.; van Koningsveld, G. A.; Legger, L.; Gruppen, H.; van Boekel, M. A. J. S.; Roozen, J. P.; Voragen, A. G. J. J. Sci. Food Agric. 2006, 86, 1225-1231.

https://doi.org/10.1002/jsfa.2473

6. Sparg, S. G.; Light, M. E.; van Staden, J. J. Ethnopharmacol. 2004, 94, 219-243.

https://doi.org/10.1016/j.jep.2004.05.016

7. Oda, K.; Matsuda, H.; Murakami, T.; Katayama, S.; Ohgitani, T.; Yoshikawa, M. Biol. Chem. 2000, 381, 6774.

https://doi.org/10.1515/BC.2000.009

8. Irma Podolak, I.; Galanty, A.; Sobolewska, D. Phytochem. Rev. 2010, 9, 425-474.

https://doi.org/10.1007/s11101-010-9183-z

9. Güçlü-Üstündağ, Ö.; Mazza, G. Crit. Rev. Food. Sci. Nutr. 2007, 47(3), 231-258.

https://doi.org/10.1080/10408390600698197

10. Attele, A.S.; Wu, J. A.; Yuan, C. S. Biochem. Pharmacol. 1999, 58, 1685-1693.

https://doi.org/10.1016/S0006-2952(99)00212-9

11. Price, K. R.; Johnson, I. T.; Fenwick, G. R. Crit. Rev. Food Sci. Nutr. 1987, 26, 27-135.

https://doi.org/10.1080/10408398709527461

12. Oleszek, W. A. J. Chromatogr. A. 2002, 967, 147-162.

https://doi.org/10.1016/S0021-9673(01)01556-4

13. Lednicer, D. Strategies for Organic Drug Synthesis, 2nd Edn.; John Wiley \& Sons: Inc.: Hoboken, New Jersey, 2009, Chapters 4 and 5 , and references therein.

14. Iglesias-Arteaga, M. A.; Pérez-Gil, R.; Pérez-Martínez, C. S.; Coll, F. J. Chem. Soc. Perkin Trans. I 2001, 261266.

https://doi.org/10.1039/b007656m

15. Iglesias-Arteaga, M. A.; Pérez-Martínez, C. S.; Coll, F. Steroids 2002, 67, 159-163.

https://doi.org/10.1016/S0039-128X(01)00150-7 
16. Romero-Ávila, M.; de Dios-Bravo, G.; Mendez-Stivalet, J. M.; Rodríguez-Sotres, R.; Iglesias-Arteaga, M. A. Steroids 2007, 72, 955-959.

https://doi.org/10.1016/..steroids.2007.08.007

17. Rosado-Abón, A.; de Dios-Bravo, G.; Rodríguez-Sotres, R.; Iglesias-Arteaga, M. A. J. Steroid Biochem Mol. Biol. 2013, 134, 45-50.

https://doi.org/10.1016/j.jsbmb.2012.10.007

18. For cephalostatins and ritterazzines see Iglesias-Arteaga, M. A.; Morzycki, J.W. In The Alkaloids: Chemistry and Biology, Vol. 72; Knölker, H.-J., Ed.; Academic Press, 2013, 153-279.

https://doi.org/10.1016/B978-0-12-407774-4.00002-9

19. For cytotoxic sterols see Xu, Q.-H.; Peng, X.-W.; Tian, W.-S. Tetrahedron Lett. 2003, 44, 9375-9377. https://doi.org/10.1016/j.tetlet.2003.09.227

20. Jiang, B.; Shi, H.; Tian, W.; Zhou, W-S. Tetrahedron 2008, 64, 469-476.

https://doi.org/10.1016/j.tet.2007.11.028

21. Sahu, N. P.; Banerjee, S.; Mondal, N. B.; Mandal, D. Steroid saponins, in Progress in the Chemistry of Organic Compounds. Kinghorns, A. D.; Falk, H.; Kobayashi, J. Eds. Springer: Wein 2008.

https://doi.org/10.1007/978-3-211-74019-4 2

22. Sparg, S. G.; Light, M.E.; van Staden, J. J. Ethnopharmacol 2004, 94, 219-243.

https://doi.org/10.1016/j.jep.2004.05.016

23. Agrawal, P. K.; Jain, D.C.; Pathak, A. K. Magn. Reson. Chem. 1995, 33, 923-953.

https://doi.org/10.1002/mrc.1260331202

24. Yang, Y.; Laval, S.; Yu. B. in Advances in Carbohydrate Chemistry and Biochemistry. Horton D. Ed. Vol. 71 Academic Press: San Diego, 2014.

25. Xiao, G.; Shao, X.; Zhou, D.; Biao, Y. Nat. Prod. Rep. 2019, 36, 769-787. https://doi.org/10.1039/C8NP00087E

26. López, Y.; Ruíz-Pérez, K. M.; Yépez, R.; Santillan, R.; Flores-Alamo, M.; Iglesias-Arteaga, M. A. Steroids2008, 73, 657-668.

https://doi.org/10.1016/j.steroids.2008.02.003

27. Barton D. H. R.; Sammes P. G.; Taylor M.V.; Werstiuk E. J Chem Soc (C) 1970, 1977-1981. https://doi.org/10.1039/j39700001977

28. Rosado-Abón, A.; Romero-Ávila, M.; Iglesias-Arteaga, M. A. Arkivoc 2010, (x), 110-115. https://doi.org/10.3998/ark.5550190.0011.a10

29. Iglesias-Arteaga, M. A.; Sandoval-Ramirez, J.; Mata-Esma, M. Y.; Viñas-Bravo, O.; Bernés, S. Tetrahedron Lett. 2004, 45, 4921-4926.

https://doi.org/10.1016/j.tetlet.2004.04.119

This paper is an open access article distributed under the terms of the Creative Commons Attribution (CC BY) license (http://creativecommons.org/licenses/by/4.0/) 